\title{
A 32-gene risk index: a new prognostic approach for prostate cancer progression
}

\author{
Chao Cai ${ }^{1,2}$, Wei-De Zhong ${ }^{3}$, W. Scott McDougal ${ }^{1}$ and Chin-Lee $\mathrm{Wu}^{1,4}$ \\ Asian Journal of Andrology (2013) 15, 590-591; doi:10.1038/aja.2013.85; published online 10 June 2013
}

$\mathbf{R}$ adical prostatectomy (RP) has been a widely accepted and standard treatment for clinically localized and locally advanced prostate cancer. However, effective clinical management of RP patient remains being challenged, given that conventional prognostic factors, including Gleason score, pT stage, surgical margin status and presurgery serum prostatespecific antigen (PSA), cannot correctly predict cancer recurrence after RP. Recently, a robust prognostic model for biochemical recurrence $(\mathrm{BCR})$ and metastasis has been developed by our group at the MGH. This model was a continuous risk index based on the expression of 32 genes and was validated in an independent, blinded cohort of 270 RP patients. In multivariate analyses, the 32-gene risk index model was prognostic for risk of BCR and had added value over the traditional parameters. This model may contribute to accurate postoperative treatment decision and hopefully help patients and doctors make a more reasonable selection at the stage of a positive biopsy.

Prostate cancer is the most common nondermatological cancer and has a second leading cause of cancer-related death in American men. In 2013, an estimated 238590 new cases will be diagnosed and 29720 men will die of this disease in United States. ${ }^{1}$ Radical prostatectomy (RP) is considered to be the first-line option for clinically localized and locally advanced prostate cancer. Early diagnosis provides an opportunity to this curative

${ }^{1}$ Department of Urology, Massachusetts Genera Hospital and Harvard Medical School, Boston, MA 02114, USA; ${ }^{2}$ Graduate School of Southern Medical University, Guangzhou, Guangdong 510515, China; ${ }^{3}$ Department of Urology, Guangzhou First Municipal People's Hospital, Guangzhou, Guangdong 510180 China and ${ }^{4}$ Department of Pathology, Massachusetts General Hospital and Harvard Medical School, Boston, MA 02114, USA

Correspondence: Dr CL Wu (cwu2@partners.org) treatment. However, approximately onethird of patients would experience biochemical recurrence $(\mathrm{BCR})$ in 10 years after $\mathrm{RP} .^{2}$ Prostate-specific antigen (PSA) is typically evaluated every 3 months after surgery, PSA should reach undetectable levels in 4 weeks in most patients after RP. ${ }^{3}$ A detectable PSA may mean undergoing BCR according to various BCR definitions in different institutes. ${ }^{4}$ BCR is accepted to be a result of histologically undetectable micrometastases in the pelvic lymph nodes at the time of RP, which implies RP patients potentially have a clinical and imaging metastasis. ${ }^{5}$ Adjuvant treatments, such as adjuvant radiotherapy, will be recommended to RP patients with adverse pathological parameters (pathological Gleason score $\geqslant 8, \mathrm{pT} 3 / \mathrm{pT} 4$ disease, positive surgical margin, positive lymph node and so on) and postoperative irradiation significantly improved biochemical progression-free survival. ${ }^{6}$ However, these commonly used prognostic factors are insufficient to accurately predict the risk of BCR after RP; $38.2 \%$ of high-risk patients did not experience BCR in a wait-and-see group followed up for a median of 10.6 years, ${ }^{6}$ which means that these patients are usually overtreated after RP. Conversely, pathologically organ-confined patients are traditionally considered to be cured by RP and thus adjuvant treatments are generally not recommended. These patients might experience BCR in 10 years. $^{7}$ Herein doctors and patients may get into a dilemma, since adjuvant radiotherapy likely has a detrimental impact on the quality of life. Thus, it is urgent to search a more correct and effective method to stratify RP patients based on the risk of BCR. In the recent published report of $\mathrm{Wu}$ et al., ${ }^{8}$ a 32 -gene risk index model was developed and validated. This 32-gene prognostic index may contribute to accurate postoperative treatment decision.
Gene expression-based risk index has been commonly used for diagnosis and predicting disease outcomes in oncological area recently. The 32-gene risk index also based on gene expression data from the real-time RT-PCR and clinical information from a discovery cohort. Then this 32-gene prognostic signature prediction ability was validated in an independent, blinded cohort of $270 \mathrm{RP}$ patients. In multivariate analysis, the 32-gene risk index was an independent predictor of BCR after RP and enhanced significant prognostic value to conventional pathological parameters. Surgical margin status was the other factor with predictive value in the multivariate analysis. These findings indicate that the 32-gene risk index may be a strong predictor for BCR. The study also showed that the predicted 10-year progression rate was similar to the actual outcome. As expected, most patients with $\mathrm{pT} 3$ or a positive surgical margin were identified as high risk by the 32 gene risk index, while this risk index also found out a low-risk subgroup from these patients and they came out no BCR events during the follow-up period. This suggests that a fraction of traditionally high-risk patients can avoid adjuvant treatment after surgery and thus, could improve their quality of life. Conversely, some patients with pT2 disease, a negative surgical margin and a Gleason score of 6, who are considered to have a low risk of BCR after RP commonly, were identified as high risk by the 32-gene risk index. This analysis implies that some conventionally low-risk patients should accept adjuvant treatment like adjuvant radiotherapy to prevent disease from progression and metastasis.

Though there are several standard clinical factors, such as preoperative serum PSA level, clinical tumor stage, biopsy Gleason score, proportion of positive cores and highest percent tumor content, to perform radical 
treatment or not often puzzles doctors and patients due to insufficient predictive ability of these parameters. The 32-gene risk index could potentially be used to assay on core needle biopsy tissue and may aid in making reasonable selections at the stage of positive biopsy.

The 32-gene risk index model may be a more effective approach to help doctors and patients make accurate decisions at preoperative and/or postoperative stage. The implication of this model in the clinical practice is still challenged by several implications mentioned in the article. ${ }^{8}$ Next, the test will need to be validated in a multi-institutional cohort or clinical trial setting. In addition, the implication of this model in the positive core biopsy stage should be further invalidated in larger follow-up studies. Finally, 32 genes involve many molecular functions, including cell proliferation, migration, cell invasion and cell cycle. The mechanisms whereby these genes involved in prostate cancer progression need to be studied. While many works need to be done in the future, hopefully this 32 -gene risk index indeed brings an exciting and intriguing news to the genitourinary oncology area.

1 Siegel R, Naishadham D, Jemal A. Cancer statistics, 2013. CA Cancer J Clin 2013; 63: 11-30.

2 Han M, Partin AW, Pound CR, Epstein JI, Walsh PC Long-term biochemical disease-free and cancerspecific survival following anatomic radical retropubic prostatectomy. The 15-year Johns Hopkins experience. Urol Clin North Am 2001; 28: 555-65.

3 Partin AW, Oesterling JE. The clinical usefulness of prostate specific antigen: update 1994. J Urol 1994. 152: 1358-68.
4 Stephenson AJ, Kattan MW, Eastham JA, Dotan ZA, Bianco FJ Jr et al. Defining biochemical recurrence of prostate cancer after radical prostatectomy: a proposal for a standardized definition. J Clin Oncol 2006; 24: 3973-8.

5 Miyake $\mathrm{H}$, Hara I, Kurahashi T, Inoue TA, Eto $\mathrm{H}$ et al. Quantitative detection of micrometastases in pelvic lymph nodes in patients with clinically localized prostate cancer by real-time reverse transcriptasePCR. Clin Cancer Res 2007; 13: 1192-7.

6 Bolla M, van Poppel H, Tombal B, Vekemans K, da Pozzo $\mathrm{L}$ et al. Postoperative radiotherapy after radical prostatectomy for high-risk prostate cancer long-term results of a randomised controlled tria (EORTC trial 22911). Lancet 2012; 380: 2018-27.

7 Psutka SP, Feldman AS, Rodin D, Olumi AF, Wu CL et al. Men with organ-confined prostate cancer and positive surgical margins develop biochemical failure at a similar rate to men with extracapsular extension. Urology 2011; 78: 121-5.

8 Wu CL, Schroeder BE, Ma XJ, Cutie CJ, Wu S et al. Development and validation of a 32-gene prognostic index for prostate cancer progression. Proc Natl Acad SCI USA 2013; 110: 6121-6. 